\title{
On the structure of the set of solutions of a Volterra integral equation in a Banach space
}

\author{
by Krzysztof Czarnowski (Gdańsk)
}

\begin{abstract}
The set of solutions of a Volterra equation in a Banach space with a Carathéodory kernel is proved to be an $\mathcal{R}_{\delta}$, in particular compact and connected. The kernel is not assumed to be uniformly continuous with respect to the unknown function and the characterization is given in terms of a $\mathrm{B}_{0}$-space of continuous functions on a noncompact domain.
\end{abstract}

1. Preliminaries and main theorem. Given a metric space $M$, we call a subset $A \subset M$ an $\mathcal{R}_{\delta^{-}}$set if there is a decreasing sequence of compact absolute retracts $A_{n} \subset M$ such that $A=\bigcap_{n=1}^{\infty} A_{n}$. It was proved by Aronszajn [2] that instead of monotonicity of the sequence $A_{n}$ it is equivalent to assume that $A \subset A_{n}$ for all $n$ and $A_{n}$ converges to $A$ in the sense of the Hausdorff metric. Using the theorem of Aronszajn it is possible to give sufficient conditions for the zero set of a map to be an $\mathcal{R}_{\delta}$. For the Banach space case see [7]; here we shall recall the $\mathrm{B}_{0}$-space version [3]. A theorem of Vidossich ([9], Theorem 2.4) could also be used to prove Theorem (2) of this paper $\left({ }^{1}\right)$.

Let $E$ be a $\mathrm{B}_{0}$-space with topology induced by a sequence of seminorms $q_{n}: E \rightarrow \mathbb{R}_{+}, n \in \mathbb{N}$. Recall that $E$, metrized by

$$
d(x, y)=\sum_{n=1}^{\infty} \frac{q_{n}(x-y)}{2^{n}\left(1+q_{n}(x-y)\right)}, \quad x, y \in E,
$$

is a complete metric space. We assume that the sequence $q_{n}$ is nondecreasing, which does not restrict generality. Let $h: \bar{\Omega} \rightarrow E, \Omega \subset E$ open, be a continuous map and $\varepsilon_{n}$ a sequence of positive reals tending to zero. A sequence of continuous maps $h_{n}: \bar{\Omega} \rightarrow E$ is an $\left\{\varepsilon_{n}\right\}$-approximation of $h$ iff $q_{n}\left(h(x)-h_{n}(x)\right) \leq \varepsilon_{n}$ for all $n$ and $x \in \bar{\Omega}$. Let $U_{n}=\left\{x \in E: q_{n}(x)<1\right\}$.

1991 Mathematics Subject Classification: 45N05, 45D05, $47 \mathrm{H} 09$.

Key words and phrases: Volterra integral equation in a Banach space, $\mathcal{R}_{\delta}$-sets.

$\left({ }^{1}\right)$ The author thanks the referee for the remark and reference. 
(1) Lemma [3]. Let $h: \bar{\Omega} \rightarrow E$ be a continuous map such that

(1.1) if $h\left(x_{n}\right) \rightarrow 0$ then the sequence $x_{n}$ contains a convergent subsequence,

(1.2) there exists an $\left\{\varepsilon_{n}\right\}$-approximation $h_{n}: \bar{\Omega} \rightarrow E$ of $h$ such that $\left.h_{n}\right|_{h_{n}^{-1}\left(\varepsilon_{n} \bar{U}_{n}\right)}: h_{n}^{-1}\left(\varepsilon_{n} \bar{U}_{n}\right) \rightarrow \varepsilon_{n} \bar{U}_{n}$ is a homeomorphism for each $n$.

Then the set $h^{-1}(0)$ is an $\mathcal{R}_{\delta}$.

In what follows $X$ will denote a Banach space, $\|\cdot\|$ the norm in $X$ and $\gamma$ a measure of noncompactness in $X$ - either that of Kuratowski or the ball (Hausdorff) measure of noncompactness (see $[4,5]$ for definitions and properties).

Let $f: \mathbb{R}_{+} \times \mathbb{R}_{+} \times X \rightarrow X$ satisfy the following assumptions:

(i) $f$ is a Carathéodory map, i.e. the map

$$
(t, x) \mapsto f(s, t, x): \mathbb{R}_{+} \times X \rightarrow X
$$

is continuous for each $s \in \mathbb{R}_{+}$and

$$
s \mapsto f(s, t, x): \mathbb{R}_{+} \rightarrow X
$$

is strongly measurable, in the Lebesgue sense, for each $(t, x) \in \mathbb{R}_{+} \times X$ (see [1] for the definition of strong measurability and its basic properties),

(ii) $\|f(s, t, x)\| \leq b(s)\|x\|+c(s)$ for all $(s, t, x) \in \mathbb{R}_{+} \times \mathbb{R}_{+} \times X$, where $b, c: \mathbb{R}_{+} \rightarrow \mathbb{R}_{0}$ are locally integrable functions,

(iii) the uniform continuity on bounded sets of each section $t \mapsto f(s, t, x)$ is uniform with respect to $x$, i.e. for any $s \in \mathbb{R}_{+}, T \in \mathbb{R}_{+}$and $\varepsilon>0$ there is a positive $\delta$ such that $\left\|f(s, t, x)-f\left(s, t^{\prime}, x\right)\right\|<\varepsilon$ for all $x \in X$ and $t, t^{\prime} \in[0, T]$ provided $\left|t-t^{\prime}\right|<\delta$,

(iv) there is a Carathéodory function $\omega: \mathbb{R}_{+} \times \mathbb{R}_{+} \times X \rightarrow \mathbb{R}_{+}$such that $\varphi(t) \equiv 0$ is the only nonnegative continuous solution of the inequality

$$
\varphi(t) \leq 2 \int_{0}^{t} \omega(s, t, \varphi(s)) d s, \quad t \geq 0,
$$

and $\gamma(f(s, t, A)) \leq \omega(s, t, \gamma(A))$ for any bounded countable $A \subset X$ and $s, t \in \mathbb{R}_{+}$.

Consider the $\mathrm{B}_{0^{-}}$-space $E=\mathcal{C}\left(\mathbb{R}_{+}, X\right)$ of continuous functions $\mathbb{R}_{+} \rightarrow X$ with seminorms $q_{n}$ defined by

$$
q_{n}(x)=\sup \{\|x(t)\|: t \in[0, n]\}, \quad n \in \mathbb{N} .
$$

The convergence in $E$ is then the uniform convergence on bounded subsets of $\mathbb{R}_{+}$. We are interested in the set of solutions of a Volterra equation

$$
x(t)=g(t)+\int_{0}^{t} f(s, t, x(s)) d s, \quad x(\cdot) \in E, t \geq 0 .
$$


(2) ThEOREM. If the kernel $f$ satisfies the assumptions (i)-(iv) and $g \in E$, then the set of solutions of equation (I) is an $\mathcal{R}_{\delta}$.

Before proceeding to the proof we shall make a few comments on our assumptions and on links to some previous results. First notice that the assumption (ii) may be replaced, with no loss of generality, by

(ii') $\|f(s, t, x)\| \leq a(s)$ for all $(s, t, x) \in \mathbb{R}_{+} \times \mathbb{R}_{+} \times X$, where $a: \mathbb{R}_{+} \rightarrow \mathbb{R}_{+}$ is locally integrable.

This is achieved in a standard way by first applying the Gronwall inequality to find a bound for the set of solutions of (I) and then appropriately modifying $f$ to make its support bounded in $x$ (by some continuous function) without changing the set of solutions of (I) and affecting the other assumptions (i), (iii) and (iv). In the following we shall be assuming (ii') rather than (ii).

Further, note that in (iii) we do not assume uniform continuity of $f$ with respect to $x$. The assumption (iii) is automatically satisfied when $f$ does not depend on $t$, or when the space $X$ is finite-dimensional (after the modification of $f$ leading to $\left.\left(\mathrm{ii}^{\prime}\right)\right)$.

Our theorem is closely linked to a theorem of Szufla ([8], Theorem 4) where the characterization of the set of solutions of the Volterra equation is given in a Banach space of continuous functions. The assumptions given there agree with our (i)-(iii) (in fact, in [8] they are given in a more general, though complicated setting), but the key assumption is that the measure of noncompactness of the $\{1 / n\}$-approximate solutions of (I) calculated in the space of continuous functions tends to zero as $n \rightarrow \infty$. This may be difficult to verify here, when we assume (iv) and do not assume uniform continuity of $f$ with respect to $x$.

2. Proof of Theorem (2). The proof proceeds through a sequence of lemmas. First define a family of maps

$$
F_{d}: E \rightarrow E, \quad\left(F_{d} x\right)(t)=\left\{\begin{array}{ll}
0, & 0 \leq t \leq d, \\
\int_{0}^{t-d} f(s, t, x(s)) d s, & t \geq d,
\end{array} \quad d \geq 0 .\right.
$$

Then (I) may be rewritten in the form

$$
x=g+F_{0} x .
$$

(3) Lemma. For any $d \geq 0$,

(3.1) if $A \subset E$ is countable, then $F_{d} A$ is a regular subset of $E$ (i.e. it is bounded and the restrictions of its elements to any bounded subset of $\mathbb{R}_{+}$ are equicontinuous functions),

(3.2) $F_{d}: E \rightarrow E$ is a continuous map. 
P r o of. We prove (3.1). Since boundedness follows directly from (ii') we concentrate on equicontinuity. Fix $T>d$ and $\varepsilon>0$. Define a family of sets

$$
\begin{aligned}
S_{\delta}=\left\{s \in[0, T-d]: \forall_{t, t^{\prime} \in[0, T]} \forall_{x(\cdot) \in A}\left|t-t^{\prime}\right|<\delta \Rightarrow\right. & \\
& \left.\left\|f(s, t, x(s))-f\left(s, t^{\prime}, x(s)\right)\right\| \leq \varepsilon\right\}, \quad \delta>0 .
\end{aligned}
$$

Since

$$
\begin{aligned}
& S_{\delta}=\bigcap_{t \in[0, T] \cap \mathbb{Q}} \bigcap_{t^{\prime} \in[0, T] \cap(t-\delta, t+\delta) \cap \mathbb{Q}} \bigcap_{x(\cdot) \in A}\{s \in[0, T-d]: \\
& \left.\left\|f(s, t, x(s))-f\left(s, t^{\prime}, x(s)\right)\right\| \leq \varepsilon\right\}
\end{aligned}
$$

(where $\mathbb{Q}$ is the set of rational numbers) and $A$ is countable, $S_{\delta}$ is measurable for any $\delta>0$. Obviously, by (iii), $S_{\delta} \nearrow[0, T-d]$ as $\delta \searrow 0$; hence if $Z_{\delta}=[0, T-d] \backslash S_{\delta}$ then the Lebesgue measure $\left|Z_{\delta}\right| \searrow 0$ as $\delta \searrow 0$. Then for any $x(\cdot) \in A$ and $d \leq t<t^{\prime} \leq T$ such that $t^{\prime}-t<\delta$ we get

$$
\begin{aligned}
\left\|\left(F_{d} x\right)\left(t^{\prime}\right)-\left(F_{d} x\right)(t)\right\| \leq & \int_{0}^{t-d}\left\|f\left(s, t^{\prime}, x(s)\right)-f(s, t, x(s))\right\| d s \\
& +\int_{t-d}^{t^{\prime}-d}\left\|f\left(s, t^{\prime}, x(s)\right)\right\| d s \\
\leq & \varepsilon(T-d)+2 \int_{Z_{\delta}} a(s) d s+\int_{t-d}^{t^{\prime}-d} a(s) d s,
\end{aligned}
$$

where the right-hand side may be made arbitrarily small by taking $\varepsilon$ and $\delta$ sufficiently small. This completes the proof of (3.1).

To prove (3.2) notice that $F_{d} E \subset E$ by (3.1). To prove continuity assume that $x_{n} \rightarrow x$ in $E$ (i.e. uniformly on each bounded subset of $\mathbb{R}_{+}$), take any $\varepsilon>0, T>d$ and this time define

$$
\begin{aligned}
S_{\delta}=\left\{s \in[0, T-d]: \forall_{t \in[0, T]} \forall_{n \in \mathbb{N}}\left\|x_{n}(s)-x(s)\right\|<\delta \Rightarrow\right. & \\
& \left.\left\|f\left(s, t, x_{n}(s)\right)-f(s, t, x(s))\right\| \leq \varepsilon\right\}, \quad \delta>0 .
\end{aligned}
$$

Again, $S_{\delta}, \delta>0$, is a family of measurable subsets of $[0, T-d]$ and $S_{\delta} \nearrow$ $[0, T-d]$ as $\delta \searrow 0$, since the continuity of $f(s, t, \cdot)$ at $x(s)$ is uniform with respect to $t \in[0, T]$. Thus $\left|Z_{\delta}\right| \searrow 0$ as $\delta \searrow 0$, where $Z_{\delta}=[0, T-d] \backslash S_{\delta}$. For a fixed $\delta>0$ we get

$$
\begin{aligned}
\left\|\left(F_{d} x_{n}\right)(t)-\left(F_{d} x\right)(t)\right\| & \leq \int_{0}^{t-d}\left\|f\left(s, t, x_{n}(s)\right)-f(s, t, x(s))\right\| d s \\
& \leq \varepsilon(T-d)+2 \int_{Z_{\delta}} a(s) d s
\end{aligned}
$$


for any $d \leq t \leq T$ and $n \in \mathbb{N}$ sufficiently large (so that $\left\|x_{n}(s)-x(s)\right\|<\delta$ for all $s \in[0, T-d])$. Since the right-hand side may be made arbitrarily small provided $\varepsilon$ and $\delta$ are taken sufficiently small, this proves that $\left(F_{d} x_{n}\right)(\cdot) \rightarrow\left(F_{d} x\right)(\cdot)$ uniformly on $[0, T]$, hence $F_{d} x_{n} \rightarrow F_{d} x$ in $E$, since $T$ is arbitrary.

In the proof of the next lemma we use the following theorem of Heinz [6].

(4) THEOREM. If $V$ is a countable set of strongly measurable functions $[0, T] \rightarrow X, T>0$, such that $\|v(s)\| \leq u(s)$ for all $v \in V$ and $s \in[0, T]$, where $u:[0, T] \rightarrow \mathbb{R}_{+}$is a fixed integrable function, then the function $s \mapsto$ $\gamma(V(s)):[0, T] \rightarrow \mathbb{R}_{+}$is integrable and

$$
\gamma\left(\int_{0}^{T} V(s) d s\right) \leq 2 \int_{0}^{T} \gamma(V(s)) d s .
$$

(5) Lemma. For any $d \geq 0$ the map $I-F_{d}: E \rightarrow E, I$ being the identity, is proper and hence also closed.

Proof. It suffices to prove that for every compact $C \subset E$ any closed and countable $A \subset\left(I-F_{d}\right)^{-1}(C)$ is compact. Since $A \subset C+F_{d} A$ it follows from Lemma (3) that $A$ is a regular subset of $E$, and hence the function

$$
\varphi: \mathbb{R}_{+} \rightarrow \mathbb{R}_{+}, \quad \varphi(t)=\gamma(A(t)),
$$

is continuous. Since $A(t) \subset C(t)+\left(F_{d} A\right)(t)$, we get

$$
\varphi(t) \leq \gamma\left(\left(F_{d} A\right)(t)\right) \text {. }
$$

If $t \geq d$, then

$$
\begin{aligned}
\varphi(t) & \leq \gamma\left(\int_{0}^{t-d} f(s, t, A(s)) d s\right) \leq 2 \int_{0}^{t-d} \gamma(f(s, t, A(s))) d s \\
& \leq 2 \int_{0}^{t-d} \omega(s, t, \varphi(s)) d s \leq 2 \int_{0}^{t} \omega(s, t, \varphi(s)) d s .
\end{aligned}
$$

Thus

$$
\varphi(t) \leq 2 \int_{0}^{t} \omega(s, t, \varphi(s)) d s, \quad t \geq 0,
$$

since $\left(F_{d} A\right)(t)=\{0\}$ for $0 \leq t \leq d$. In the above calculations we have applied Theorem (4) to the set of strongly measurable functions $V=f(\cdot, t, A(\cdot))$ bounded by the function $a$ from assumption ( $\left.\mathrm{ii}^{\prime}\right)$.

Assumption (iv) implies that the section $A(t)$ is compact for any $t \in \mathbb{R}_{+}$. Thus the compactness of $A$ follows from the Arzelà-Ascoli theorem.

(6) Lemma. For any $d>0$ the map $I-F_{d}: E \rightarrow E$ is a homeomorphism. 
Proof. We prove that $I-F_{d}: E \rightarrow E$ is a one-to-one map; the continuity of $\left(I-F_{d}\right)^{-1}$ then follows from (5). Let $x_{1}-F_{d} x_{1}=x_{2}-F_{d} x_{2}$ for some $x_{1}, x_{2} \in E$. Then $x_{1}-x_{2}=F_{d} x_{1}-F_{d} x_{2}$, which means that

$$
x_{1}(t)-x_{2}(t)=\left\{\begin{array}{l}
0, \quad 0 \leq t \leq d, \\
\int_{0}^{t-d}\left(f\left(s, t, x_{1}(s)\right)-f\left(s, t, x_{2}(s)\right)\right) d s, \quad t \geq d .
\end{array}\right.
$$

It easily follows that $x_{1}(t)=x_{2}(t)$ for all $t \in \mathbb{R}_{+}$, that is, $x_{1}=x_{2}$. Now, given $y \in E$, define $x \in E$ by

$$
x(t)=\left\{\begin{array}{l}
y(t), \quad 0 \leq t \leq d, \\
y(t)+\int_{0}^{t-d} f(s, t, x(s)) d s, \quad t \geq d .
\end{array}\right.
$$

Obviously $\left(I-F_{d}\right) x=y$, which completes the proof.

(7) Lemma. For any fixed $n \in \mathbb{N}$,

$$
\lim _{d \rightarrow 0} \sup _{x \in E} q_{n}\left(F_{d} x-F_{0} x\right)=0 .
$$

Proof. Since

$$
\left(F_{0} x\right)(t)-\left(F_{d} x\right)(t)= \begin{cases}\int_{0}^{t} f(s, t, x(s)) d s, & 0 \leq t \leq d, \\ 0 & t \\ \int_{t-d}^{t} f(s, t, x(s)) d s, & t \geq d\end{cases}
$$

we have $\left\|\left(F_{0} x\right)(t)-\left(F_{d} x\right)(t)\right\| \leq \int_{\mathbb{R}_{+} \cap[t-d, t]} a(s) d s$ and the assertion follows from integrability of $a(\cdot)$ over $[0, n]$ for all $n$.

To finish the proof of the theorem take any sequence $d_{n} \searrow 0$, define

$$
\begin{aligned}
h: E \rightarrow E, \quad h(x) & =x-F_{0} x-g, \\
h_{n}: E \rightarrow E, \quad h_{n}(x) & =x-F_{d_{n}} x-g,
\end{aligned}
$$

and notice that all the assumptions of Lemma (1) are satisfied. Hence the set $h^{-1}(0)$, which coincides with the set of solutions of equation (I), is an $\mathcal{R}_{\delta}$.

\section{References}

[1] A. Alexiewicz, Functional Analysis, PWN, Warszawa, 1969 (in Polish).

[2] N. Aronszajn, Le correspondant topologique de l'unicité dans la théorie des équations différentielles, Ann. of Math. 43 (1942), 730-738. 
[3] K. Czarnowski and T. Pruszko, On the structure of fixed point sets of compact maps in $\mathrm{B}_{0}$ spaces with applications to integral and differential equations in unbounded domain, J. Math. Anal. Appl. 154 (1991), 151-163.

[4] K. Deimling, Ordinary Differential Equations in Banach Spaces, Lecture Notes in Math. 596, Springer, Berlin, 1977.

[5] K. Goebel, Thickness of sets in metric spaces and applications in fixed point theory, habilitation thesis, Lublin, 1970 (in Polish).

[6] H. P. Heinz, On the behaviour of measures of noncompactness with respect to differentiation and integration of vector-valued functions, Nonlinear Anal. 7 (1983), 1351-1371.

[7] J. M. Lasry et R. Robert, Analyse non linéaire multivoque, Centre de Recherche de Math. de la Décision, No. 7611, Université de Paris-Dauphine.

[8] S. Szufla, On the structure of solution sets of differential and integral equations in Banach spaces, Ann. Polon. Math. 34 (1977), 165-177.

[9] G. Vidossich, On the structure of the set of solutions of nonlinear equations, J. Math. Anal. Appl. 34 (1971), 602-617.

INSTITUTE OF MATHEMATICS

UNIVERSITY OF GDAŃSK

WITA STWOSZA 57

80-952 GDAŃSK, POLAND 